\title{
The Design of a comparative amplifier for deep sea
}

\author{
Xie Yongchao Shi Jinyan \\ (Hunan Railway Professional Technology College, ZhuZhou HN412001, P. R. China) \\ Xieyongchao2008@126.com
}

Key words: Comparison amplifier; Differential amplifier; Design

Abstract: By the advantage of high CMRR and high input impedance of the parallel connected differential amplifier, this paper designs a comparison amplifier which has good linearity.

\section{Introduction}

Since 1960s, many developed countries have taken the lead in marching into the deep-sea ocean, which has promoted the rapid development of deep sea exploration technology. In twenty-first Century, all countries in the world were strengthening the development of the ocean. The technology of drilling technology, such as drilling rig, detecting instrument and equipment, underwater vehicle, deep submersible, sampling equipment and so on, has been developing constantly, and the depth of marine exploration technology is constantly being refreshed. Seawater pressure is a key problem in marine exploration. Therefore, dynamic pressure compensation is needed to solve deep-sea seawater pressure problems for deep-sea exploration.

Because the proportional amplifier controls the closed-loop electro-hydraulic proportional variable pump by using relative working pressure signal, the electrical compensation control method is applied to eliminate the superimposed seawater pressure. The two pressure sensors placed in the pressure tank were used to detect the absolute working pressure of the system and the seawater environment pressure, using the comparison amplifying circuit can be two pressure signal linear operation is transformed into a pressure signal input proportional amplifier, so as to adapt to the characteristics of input interface, proportion control amplifier.

The main function of the comparative amplifier is to perform linear operation on the two way pressure feedback signal and make its output signal satisfy the input requirement of the proportional amplifier. Therefore, the comparative amplifier should have high (CMRR), high input impedance and better linearity.

\section{The design principle of the comparative amplifier}

The comparison amplifier designed in this paper is composed of a matched amplifier, a subtracer, a filter and a power supply, and the working principle diagram is shown in Figure 1. The two pressure sensors in Figure 1, respectively, $\mathrm{P}_{1}$ detect the outlet pressure of the variable pump, and $\mathrm{P}_{2}$ detect the pressure of the sea water. The detection signals of the two sensors are passed through the matched amplifier into the subtracer for linear matching, then passed the filter, and finally sent to the proportional amplifier. 


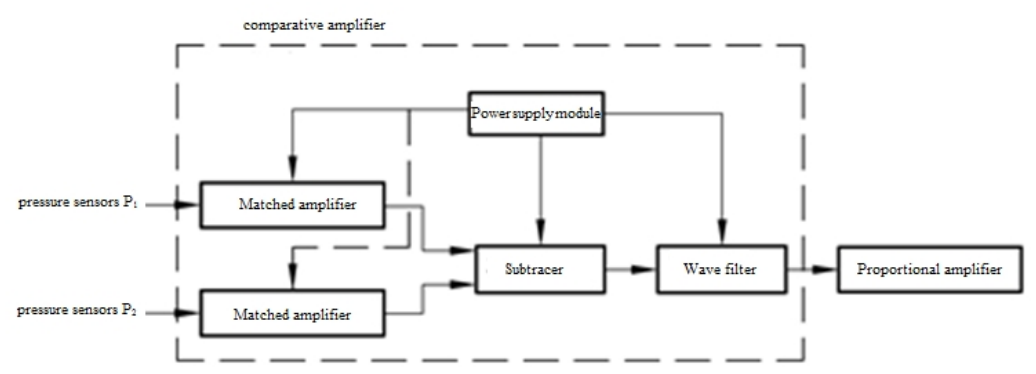

Fig.1 working principle block diagram of comparison amplifier

\section{Matched amplifier}

Since the output signal of the detection element can not be directly used as a feedback signal, it is necessary to the further processing of the matched amplifier to make the comparison between the feedback signal and the given signal accord with the actual conditions, and play a buffer role, which can increase the conversion accuracy of the system.

In essence, the matched amplifier is the phase and reverse phase amplification circuit, and the design in this paper uses a reverse phase amplifier, as shown in Fig.2. The operational amplifier U1 and its peripheral devices constitute a matched amplifier. The tuning of the given conditions is realized by $\mathrm{R} 41$ and $\mathrm{R} 44$. The resistance $\mathrm{R} 41$ is zeroing resistance. If the input signal is zero, adjust the R41 to make the output zero. The resistance R44 is amplitude modulation resistance. If the maximum input value is given, then R44 is adjusted to maximize the output.

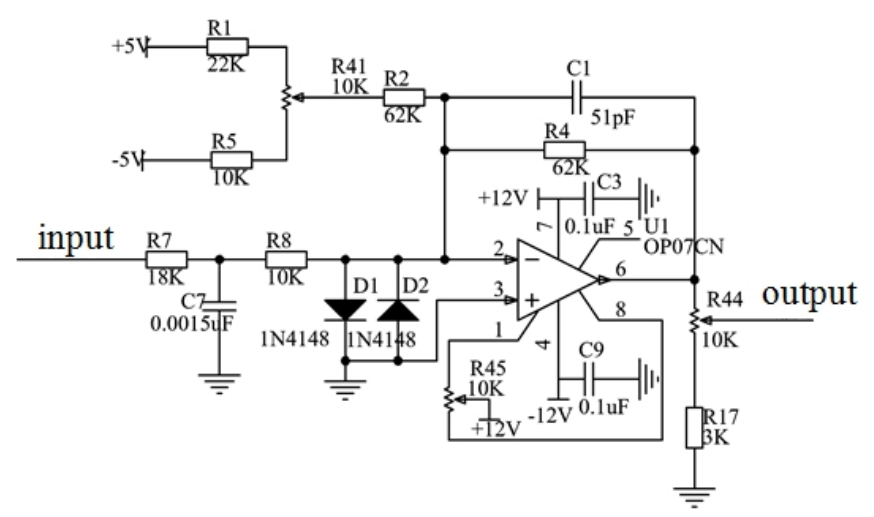

Fig. 2 matched amplifier

\section{Subtracer}

A subtracer is a differential operational amplifier composed of an operational amplifier, which makes the output signal of the matched amplifier to a certain proportion. The differential amplifier can amplify the differential mode signal and suppress the common mode signal, which requires high input impedance and high CMRR. There are two factors that influence the CMRR of the basic differential amplifier: (1) the matching accuracy of the external resistance of the circuit; (2) the size of CMRR of amplifier itself.

The basic differential amplifier with low input impedance, CMRR is not high, in order to solve the problem, the design adopts three differential amplifier composed of amplifying circuit, as shown in Fig. 3, U2, U6 and its peripheral circuit phase parallel differential amplifier, U8 and peripheral circuit basic differential amplifier. In order to achieve higher CMRR, the design uses operational amplifier OP07 with very low offset voltage, very low offset voltage, temperature drift, very low input voltage, noise amplitude and high CMRR. 


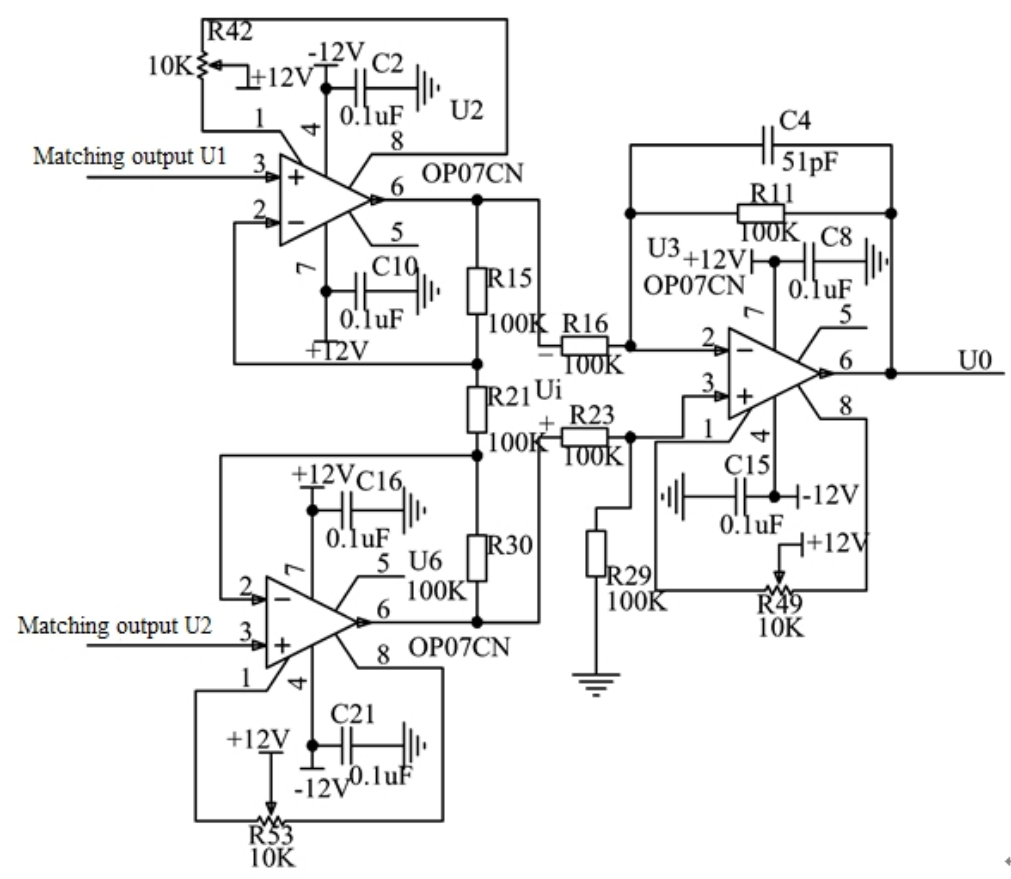

Fig. 3 Subtracer

According to the principle of superposition, the input signal $\mathrm{U}_{1}$ and $\mathrm{U}_{2}$ are decomposition combined into input differential mode $\mathrm{U}_{\mathrm{d}}$ and common mode signal $\mathrm{U}_{\mathrm{c}}$, if the component $\mathrm{U}_{0 \mathrm{~d}}$ and $\mathrm{U}_{0 \mathrm{c}}$ produced by the total output $\mathrm{U}_{0}$ of the circuit, it is known that

$$
U_{0 d}=\left(1+\frac{R_{15}+R_{30}}{R_{21}}\right) \frac{R_{11}}{R_{16}} U_{d}=K_{f 26} K_{f 8} U_{d}
$$

In the formula, $K_{f 26}$ is the differential gain of the intermediate phase parallel differential amplifier; $K_{f 8}$ is the differential gain of the basic differential amplifier.

The total difference mode gain is:

$$
K_{f}=K_{f 26} K_{f 8}
$$

In order to better suppress the signals of common mode and misalignment, an external circuit with symmetric parameters is used, taking $R_{15}=R_{30}=R_{11}=R_{f}$, the total difference mode gain at this time is as follows:

$$
K_{f}=\left(1+\frac{2 R_{f}}{R_{21}}\right) K_{f 8}
$$

Because the operational amplifier U2 and U6 transmit the common mode input signal Uc to its output end at 1: 1, that is, the common mode input voltage $\mathrm{Uc}$ is for $\mathrm{U} 8$, then the total common mode gain is

$$
U_{0 c}=K_{f c 8} U_{c}, K_{f c}=K_{f c 8}
$$

The total CMRR of the circuit is

$$
C M R R_{\Sigma}=\frac{K_{f}}{K_{f c}}=\frac{K_{f 26} K_{f 8}}{K_{f c 8}}=K_{f 26} \bullet C M R R_{8}
$$

In the formula, $C M M R_{8}$ is the CMRR of the operational amplifier U8. 
The advantages of comparison amplifier are: high input impedance, change the size of $\mathrm{R}_{21}$ can adjust the gain; the external circuit does not need matching resistors, and a high CMRR can be obtained. A basic differential amplifier is connected to a phase parallel differential amplifier, which suppresses the common mode signal at the two ends of the output $\mathrm{U}_{\mathrm{i}}$ of the phase parallel differential amplifier. From the formula (5), we can see that the total differential mode gain of the comparator amplifier is increased $K_{f 26}$ multiple campared with the basic differential amplifier circuit, and the total CMRR is also increased $K_{f 26}$ multiple campared with the basic differential amplifier $\mathrm{CMMR}_{8}$.

\section{Conclusion}

A design of comparative amplifier for deep sea is presented in this paper. The comparison amplifier has good linearity, made of, by using the advantages of high CMRR and high input impedance of the phase parallel differential amplifier.

\section{Acknowledgment}

This work was supported by A Project Supported by Scientific Research Fund of Hunan Provincial Education Department (No. 17B179)

\section{References}

[1] Kang Huaguang. Analog part of electronic technology [M]. Beijing: Higher Education Press, 2000

[2] Yan Meihui, Yang Guangbi. Analysis and application of integrated operational amplifier[M]. Chengdu: University of Electronic Science and technology, 1992

[3] Wu Genmao. A new practical electro-hydraulic proportional technology[M]. Hangzhou: Zhejiang University press, 2006

[4] Yin Jun. Application of proportional amplifier in hydraulic system [J]. Tianjin metallurgy, 2002,108 (3)

[5] Wu Shuikang, Fu Hanguang. Proportional amplifier with different impedance [J].Hydraulic pneumatic and seal, 2002,92 (2) 